University of Wollongong

Research Online

Australian Institute for Innovative Materials -

Papers

Australian Institute for Innovative Materials

$1-1-2020$

Atomic-scale origin of ultrahigh piezoelectricity in samarium-doped PMN-

PT ceramics

Chunchun Li

Bin Xu

Dabin Lin

Shujun Zhang

University of Wollongong, shujun@uow.edu.au

Laurent Bellaiche

See next page for additional authors

Follow this and additional works at: https://ro.uow.edu.au/aiimpapers

Part of the Engineering Commons, and the Physical Sciences and Mathematics Commons

Research Online is the open access institutional repository for the University of Wollongong. For further information contact the UOW Library: research-pubs@uow.edu.au 


\title{
Atomic-scale origin of ultrahigh piezoelectricity in samarium-doped PMN-PT ceramics
}

\author{
Abstract \\ Designing high-performance piezoelectric materials based on atomic-scale calculations is highly desired \\ in recent years, following the understanding of the structure-property relationship of state-of-the-art \\ piezoelectric materials. Previous mesoscale simulations showed that local structural heterogeneity plays \\ an important role in the piezoelectric property of ferroelectrics; that is, larger structural heterogeneity \\ leads to higher piezoelectricity. In this Rapid Communication, by combining first-principles calculations \\ and experimental characterizations, we explored the atomic-scale origin of the high piezoelectricity for \\ samarium-doped $\mathrm{Pb}(\mathrm{Mg} 1 / 3 \mathrm{Nb} 2 / 3)$ O3-PbTiO3 (PMN-PT) ceramics, which possesses the highest \\ piezoelectric d33 of $\sim 1500 \mathrm{pCN}-1$ among all known piezoelectric ceramics. The impacts of various \\ dopants on local structure and piezoelectric properties of PMN-PT ceramics were investigated in terms of \\ the effective ionic radius and cation valence. Our results show that A-site dopants with a valence of 3+ \\ are more effective to produce local structural heterogeneity in PMN-PT when compared with the A-site \\ dopants with a valence of $2+$, and a smaller dopant size leads to a larger variation of local structure. \\ According to this study, the outstanding piezoelectricity in Sm-doped PMN-PT ceramics is attributed to \\ the fact that $\mathrm{Sm3}+$ is the smallest ions that can entirely go to the A site of PMN-PT rather than the B site. \\ The present work may benefit the design of high-performance piezoelectric materials based on the \\ concept of local structural engineering. \\ Disciplines \\ Engineering | Physical Sciences and Mathematics

\section{Publication Details} \\ Li, C., Xu, B., Lin, D., Zhang, S., Bellaiche, L., Shrout, T. R. \& Li, F. (2020). Atomic-scale origin of ultrahigh \\ piezoelectricity in samarium-doped PMN-PT ceramics. Physical Review B, 101 (14), 140102-1-140102-7.

\section{Authors} \\ Chunchun Li, Bin Xu, Dabin Lin, Shujun Zhang, Laurent Bellaiche, Thomas R. Shrout, and Fei Li
}




\title{
Atomic-scale origin of ultrahigh piezoelectricity in samarium-doped PMN-PT ceramics
}

\author{
Chunchun Li $\odot,{ }^{1,2}$ Bin Xu $\odot,{ }^{3,4, *}$ Dabin Lin, ${ }^{2}$ Shujun Zhang $\odot,{ }^{5}$ Laurent Bellaiche, ${ }^{4}$ Thomas R. Shrout, ${ }^{2}$ and Fei Li $\odot^{2,6, \dagger}$ \\ ${ }^{1}$ College of Information Science and Engineering, Guilin University of Technology, Guilin 541004, China \\ ${ }^{2}$ Materials Research Institute, Pennsylvania State University, University Park, State College, Pennsylvania 168001, USA \\ ${ }^{3}$ School of Physical Science and Technology, Soochow University, Suzhou, Jiangsu 215006, China \\ ${ }^{4}$ Natural Science Department of Physics, University of Arkansas, Fayetteville, Arkansas 72701, USA \\ ${ }^{5}$ Institute for Superconducting and Electronic Materials, Australian Institute for Innovative Materials, University of Wollongong, \\ Wollongong New South Wales 2500, Australia \\ ${ }^{6}$ Electronic Materials Research Laboratory, Key Laboratory of the Ministry of Education and International Center for Dielectric Research, \\ Xi'an Jiaotong University, Xi'an 710049, China
}

(Received 5 July 2019; revised manuscript received 31 March 2020; accepted 1 April 2020; published 27 April 2020)

\begin{abstract}
Designing high-performance piezoelectric materials based on atomic-scale calculations is highly desired in recent years, following the understanding of the structure-property relationship of state-of-the-art piezoelectric materials. Previous mesoscale simulations showed that local structural heterogeneity plays an important role in the piezoelectric property of ferroelectrics; that is, larger structural heterogeneity leads to higher piezoelectricity. In this Rapid Communication, by combining first-principles calculations and experimental characterizations, we explored the atomic-scale origin of the high piezoelectricity for samarium-doped $\mathrm{Pb}\left(\mathrm{Mg}_{1 / 3} \mathrm{Nb}_{2 / 3}\right) \mathrm{O}_{3}-\mathrm{PbTiO}_{3}$ (PMN-PT) ceramics, which possesses the highest piezoelectric $d_{33}$ of $\sim 1500 \mathrm{pC} \mathrm{N}^{-1}$ among all known piezoelectric ceramics. The impacts of various dopants on local structure and piezoelectric properties of PMN-PT ceramics were investigated in terms of the effective ionic radius and cation valence. Our results show that $A$-site dopants with a valence of $3+$ are more effective to produce local structural heterogeneity in PMN-PT when compared with the $A$-site dopants with a valence of $2+$, and a smaller dopant size leads to a larger variation of local structure. According to this study, the outstanding piezoelectricity in Sm-doped PMN-PT ceramics is attributed to the fact that $\mathrm{Sm}^{3+}$ is the smallest ions that can entirely go to the $A$ site of PMN-PT rather than the $B$ site. The present work may benefit the design of high-performance piezoelectric materials based on the concept of local structural engineering.
\end{abstract}

DOI: $10.1103 /$ PhysRevB.101.140102

\section{INTRODUCTION}

Piezoelectric materials, which can generate electric fields/charges by subjecting to a mechanical force, or exhibit a mechanical strain when they are under an electrical field, are essential for numerous applications, e.g., ultrasonic medical imaging, underwater acoustics and actuators, etc. [1-3]. Among all known piezoelectrics, perovskite ferroelectrics exhibit the highest piezoelectric charge coefficients and electromechanical coupling factors, thus they have been widely studied and utilized for piezoelectric devices [4-12]. Over the past several decades, morphotropic phase boundary (MPB) was thought to be a critical factor to achieve high piezoelectricity, and the exploration of new perovskite ferroelectrics has generally been focused on the design of new MPBs [13-17]. However, this approach has reached a plateau after the development of over 60 years, and alternative design strategies are required to further improve the piezoelectric performance. Based on the investigations of relaxor ferroelectrics, it has

\footnotetext{
*Corresponding author: binxu19@ @uda.edu.cn

†Corresponding author: ful5@xjtu.edu.cn
}

been proposed that local structural heterogeneity can have a great impact on the piezoelectric property [18-25]. Specifically, by mesoscale phase-field simulations, Li et al. showed that the structural heterogeneity can lead to additional interfacial energies, including electrostatic, elastic, and gradient energies, as a result of polarization and strain discontinuities. The competition between the bulk and interfacial energies may give rise to a flattened free-energy profile and enhanced piezoelectricity [18].

Very recently, samarium (Sm) dopants were found to have an ability to further enhance the local structural heterogeneity of $\mathrm{Pb}\left(\mathrm{Mg}_{1 / 3} \mathrm{Nb}_{2 / 3}\right) \mathrm{O}_{3}-\mathrm{PbTiO}_{3}$ (PMN-PT) perovskites. In Sm-doped PMN-PT ceramics and crystals, the piezoelectric coefficients $d_{33}$ were found to be up to 1500 and $4100 \mathrm{pC} \mathrm{N}^{-1}$, respectively, which are about twice that of pure PMN-PTs $[22,25]$. However, the atomic-scale origin of the highly enhanced structural heterogeneity and piezoelectricity for Smdoped PMN-PT has not yet been clarified, which limits the usage of local structural heterogeneity to other ferroelectric systems to achieve the enhanced piezoelectricity. To address this issue, here, with a combination of first-principles calculations and experimental characterizations, we studied the impacts of various dopants on the local structure and piezoelectric properties of PMN-PT. 
(a)

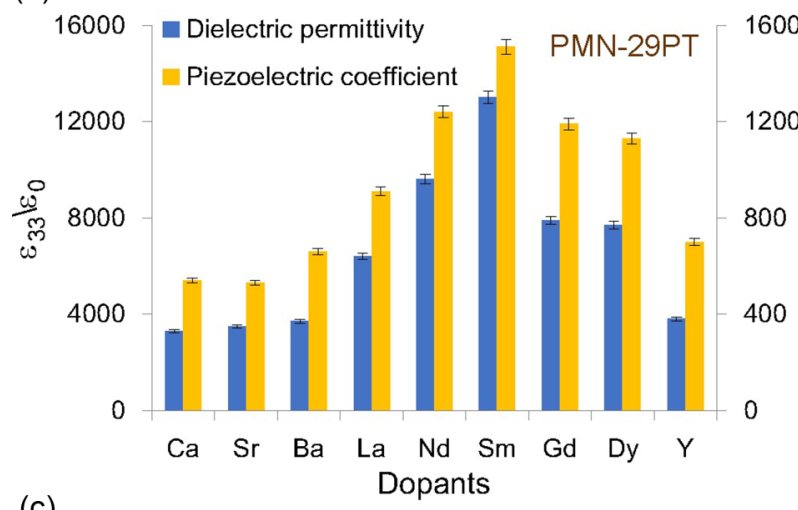

(c)

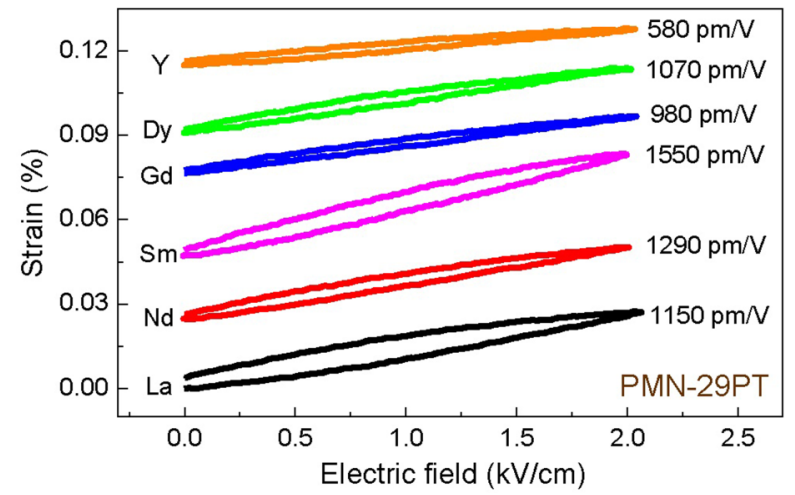

(b)
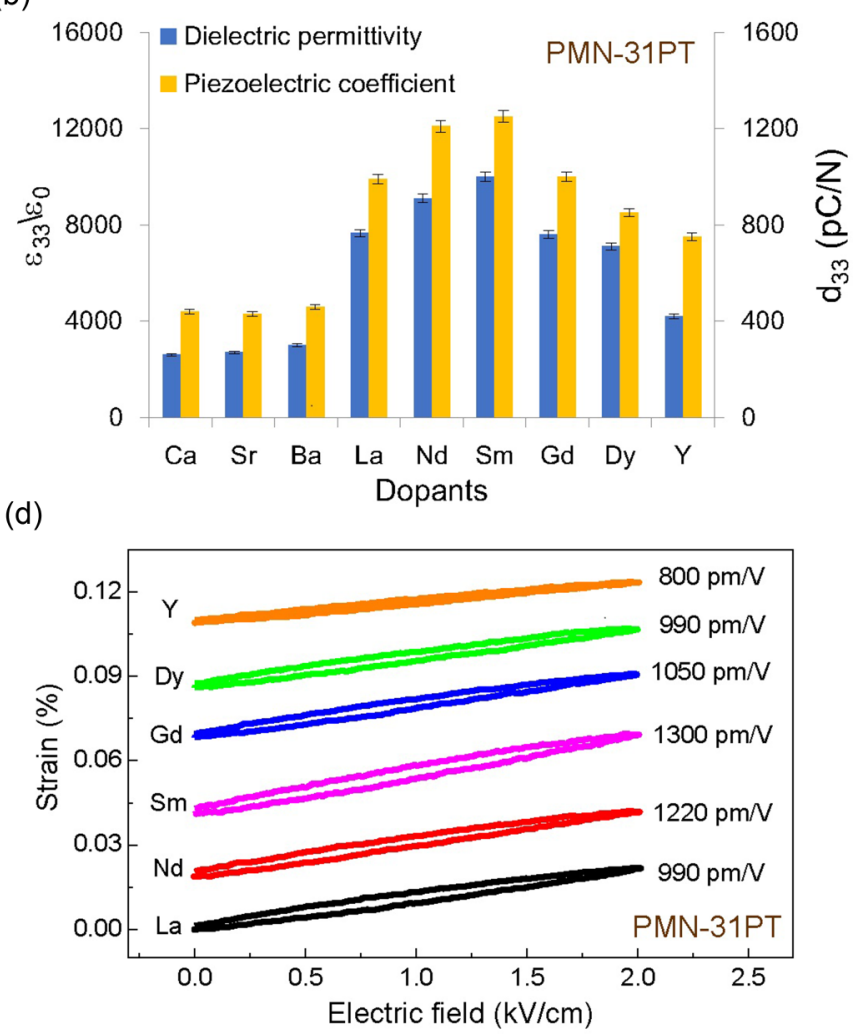

FIG. 1. Room-temperature piezoelectric coefficients and dielectric permittivities for $2.5 \mathrm{~mol} \% R$-doped (a) PMN-29PT and (b) PMN31PT; electrical-field-induced strain curves for 2.5 mol \% R-doped (c) PMN-29PT and (d) PMN-31PT.

\section{RESULTS}

The PMN-PT ceramics with various dopants were prepared by a solid-state sintering method. We focused on the 2.5 mol \% RE (rare-earth)-doped PMN-29PT and PMN31PT compositions, since the maximum piezoelectric properties are present for the PT content around $29 \%-31 \%$. The $\mathrm{Ca}-, \mathrm{Ba}-$, and Sr-doped PMN-PT ceramics were also investigated to be compared with RE-doped PMN-PT. Figure 1(a) shows the measured dielectric and piezoelectric properties, and Fig. 1(b) shows the electrical-field-induced strain curves for 2.5 mol \% RE-doped PMN-29PT and PMN-31PT, from which a large-signal piezoelectric coefficient $\left(d_{33}^{*}\right)$ can be obtained. It was observed that aliovalent +3 dopants lead to a significant increase in piezoelectric coefficients $\left(d_{33}=700-1500 \mathrm{pC} \mathrm{N}^{-1}\right.$; for comparison, $d_{33}$ of PMN29PT is $360 \mathrm{pC} \mathrm{N}^{-1}$ and dielectric permittivities $\left(\varepsilon_{33} / \varepsilon_{0}=\right.$ $4000-13$ 000; for comparison, $\varepsilon_{33} / \varepsilon_{0}$ of PMN-29PT is 2600), while isovalent dopants only lead to minimal improvements in dielectric permittivities $\left(\varepsilon_{33} / \varepsilon_{0}=2500-4000\right)$ and piezoelectric coefficients $\left(d_{33}=440-660 \mathrm{pC} \mathrm{N}^{-1}\right)$. It is interesting to note that for RE-doped PMN-PT, both dielectric permittivity and piezoelectric coefficient increase with decreasing ionic radius from $\mathrm{La}^{3+}$ to $\mathrm{Sm}^{3+}$; by further decreasing the ionic radius from $\mathrm{Sm}^{3+}$ to $\mathrm{Y}^{3+}$, however, both the values decrease, with the maxima observed in Sm-PMN-PT, as shown in Fig. 1. To explore the reason why Sm-doped PMN-PT ceramics possess the highest piezoelectricity among all doped
PMN-PT counterparts, we experimentally characterized the spontaneous polarization, microstructures, and temperaturedependent dielectric properties of PMN-31PT ceramics with various dopants.

Figures S1 and S2 in the Supplemental Material (SM) give the scanning electron microscope (SEM) images and the densities for doped PMN-PT ceramics, where no distinct difference was observed in terms of grain size (3.5$5.0 \mu \mathrm{m}$ ) and density (all samples exhibit high bulk densities of $7.8-7.82 \mathrm{~g} / \mathrm{cm}^{3}$, with relative density $>95 \%$ ) [26]. This reveals that the impacts of density and grain size on the dielectric and piezoelectric properties can be ruled out. In addition, the difference in domain size was thought to be minimal, since the domain size is closely correlated to the grain size [domain size $\propto$ (grain size $)^{m}$ ] with $m$ as an exponent, as reported in Ref. [27]. Based on the above discussion, the difference in the contributions from phase structure, density, grain size, and domain walls are minimum for different rareearth element doped PMN-PT ceramics. Figure S3 in the SM shows the polarization-vs-electric-field curves for doped ceramics, where no significant difference in $P_{r}$ values is observed for different $\mathrm{Re}^{3+}$ dopants. In addition, the electrostrictive coefficient $Q_{33}$, which is dominantly associated with the ordering degree of $B$-site cations for a PMN-PT system [28], is found to be insensitive to the small amount of dopants, as shown in Fig. S4 in the SM [26]. These results indicate that the difference in piezoelectric coefficients $d_{33}$ of RE-doped PMN-PT ceramics is attributed to the dielectric permittivity 
(a)

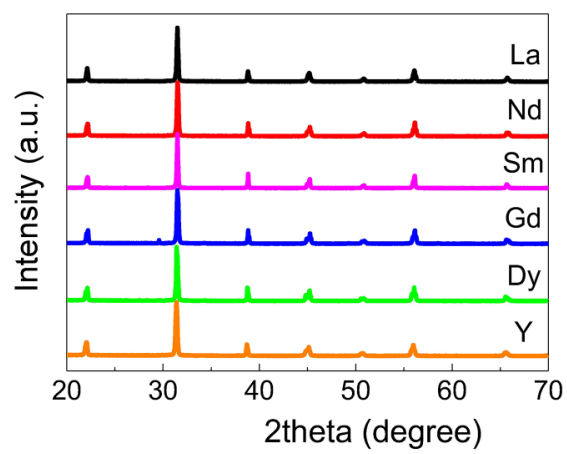

(c)

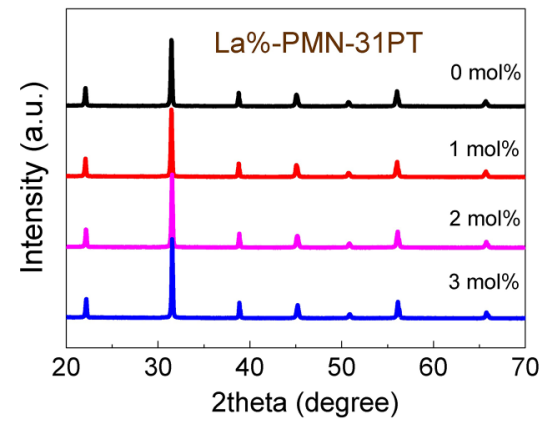

(d)

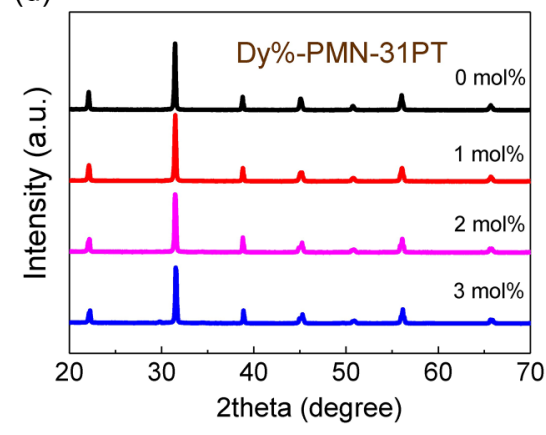

(b)

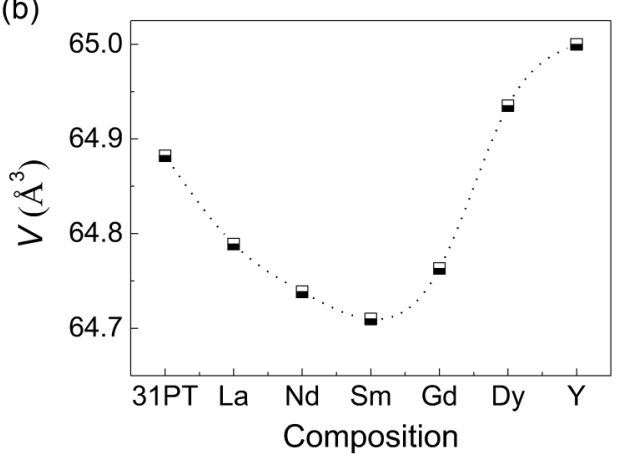

(e)

FIG. 2. (a) XRD patterns of $2.5 \mathrm{~mol} \% R$-PMN-31PT, and enlarged peak around $2 \theta=29.6^{\circ}$ for clarifying the diffraction peak of pyrochlore phase; (b) variation in cell volume as a function of composition; (c), (d) Room-temperature XRD profiles for La- and Dy-doped PMN-31PT with respect to doping levels; (e) variation in the unit cell volume of PMN-31PT with various dopants $\left(\mathrm{La}^{3+}, \mathrm{Sm}^{3+}, \mathrm{Dy}^{3+}\right.$, and $\left.\mathrm{Y}^{3+}\right)$ as a function of doping content.

variations according to $d_{33}=2 P_{r} \varepsilon_{33} Q_{33}$. From phenomenological theory, the intrinsic dielectric permittivity is associated with the curvature of the free-energy profile of a ferroelectric, i.e., a lower curvature of the free-energy profile corresponds to a larger dielectric permittivity. In our previous works by phase-field simulations, we proposed a mesoscale mechanism to explain the flattened free-energy profile for doped PMN-PT systems, i.e., a competition between the interfacial and bulk energies can result in a flattened free-energy profile, where the additional interfacial energies arise from the enhanced local structural heterogeneity [22]. In the present work, we intend to understand why Sm is the most effective dopant for inducing local structural heterogeneity in PMN-PT by density functional theory (DFT) calculations.

The location of the dopants ( $A$ or $B$ site in PMN-PT) in DFT calculations are first characterized by experiments, since it is difficult to unambiguously predict the occupational preference of the dopants by DFT without detailed information on the oxygen partial pressure and Fermi level [29]. We performed three types of experiments to study the occupational preference of $\mathrm{Re}^{3+}$ dopants, i.e., the measurements on lattice parameters, Curie temperatures, and volatilization characteristic of a $\mathrm{Pb}^{2+}$ cation. Figure 2(a) shows the $\mathrm{x}$-ray diffraction (XRD) patterns of RE-doped PMN-31PT powders, by which we verified that no macroscopic structural difference was induced by various $R^{3+}$ dopants, suggesting rare-earth elements doping mainly leads to local structural variations. By Rietveld refinements (Figs. S5 and S6), the volumes of the primary lattice are determined and given in Fig. 2(b). For the dopants of $\mathrm{La}^{3+}, \mathrm{Nd}^{3+}$, and $\mathrm{Sm}^{3+}$, the volumes of $2.5 R^{3+}$-PMN-31PT are found to be smaller than that of PMN-31PT and gradually decrease with decreasing the ionic radius from $\mathrm{Pb}^{2+}(1.49 \AA$, $\mathrm{CN}=12)$, via $\mathrm{La}^{3+}(1.36 \AA, \mathrm{CN}=12)$ and $\mathrm{Nd}^{3+}(1.27 \AA$, $\mathrm{CN}=12)$ to $\mathrm{Sm}^{3+}(1.24 \AA, \mathrm{CN}=12)$. This is only possible if the dopants enter the $A$ site of PMN-PT, i.e., substitution of $\mathrm{Pb}^{2+}$. On the contrary, by further decreasing the dopant size, the lattice is observed to expand, as shown in Fig. 2(b) $\left(\mathrm{Gd}^{3+}, \mathrm{Dy}^{3+}\right.$, and $\left.\mathrm{Y}^{3+}\right)$. This implies that the dopants $\mathrm{Gd}^{3+}$, $\mathrm{Dy}^{3+}$, and $\mathrm{Y}^{3+}$ can go to the $B$ site of PMN-PT, i.e., substitution of $\mathrm{Mg}^{2+}(0.72 \AA, \mathrm{CN}=6), \mathrm{Nb}^{5+}(0.64 \AA, \mathrm{CN}=6)$, or $\mathrm{Ti}^{4+}(0.605 \AA, \mathrm{CN}=6)$, whose ionic sizes are smaller than those of $\mathrm{Gd}^{3+}(0.938 \AA, \mathrm{CN}=6), \mathrm{Dy}^{3+}(0.912 \AA$, $\mathrm{CN}=6)$, and $\mathrm{Y}^{3+}(0.90 \AA, \mathrm{CN}=6)$.

We also studied the volume variations for doped PMN$31 \mathrm{PT}$ as a function of doping concentration, as shown in Figs. 2(c)-2(e). For $\mathrm{La}^{3+}$ and $\mathrm{Sm}^{3+}$, a nearly linear decrease of the volume is observed with increasing doping concentration. This indicates that $\mathrm{La}^{3+}$ or $\mathrm{Sm}^{3+}$ replaces the larger $\mathrm{Pb}^{2+}$ cations in PMN-PT, and $3 \mathrm{~mol} \%$ of the dopant is below the solid solution limitation without introducing impurity. In contrast, the lattice volume is found to increase linearly by increasing the concentration of $\mathrm{Dy}^{3+}$ or $\mathrm{Y}^{3+}$, which indicates that $\mathrm{Dy}^{3+} / \mathrm{Y}^{3+}$ dopants, or at least part of them, go to the $B$ site of PMN-PT, due to the fact that the ionic size of $\mathrm{Dy}^{3+} / \mathrm{Y}^{3+}(0.912 \AA / 0.90 \AA)$ is much larger than those of the $B$-site cations in PMN-PT, i.e., $\mathrm{Mg}^{2+}(0.72 \AA), \mathrm{Nb}^{5+}(0.64 \AA)$, and $\mathrm{Ti}^{4+}(0.605 \AA)$.

The variation of Curie temperature can also provide evidence for occupational preference of $R^{3+}$ dopants. According to the Landau theory [30,31], Curie temperature $\left(T_{C}\right)$ 
(a)

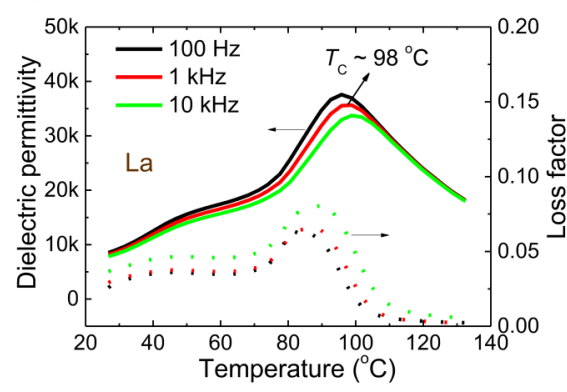

(d)

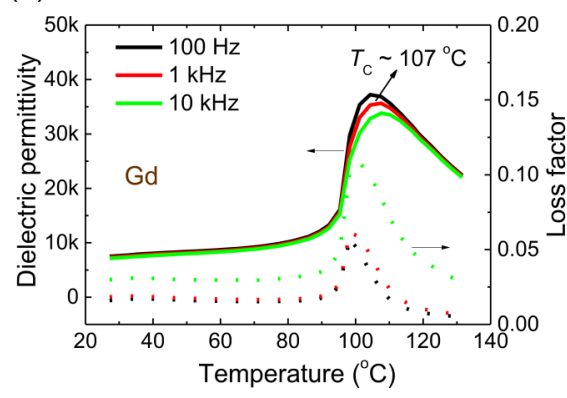

(b)

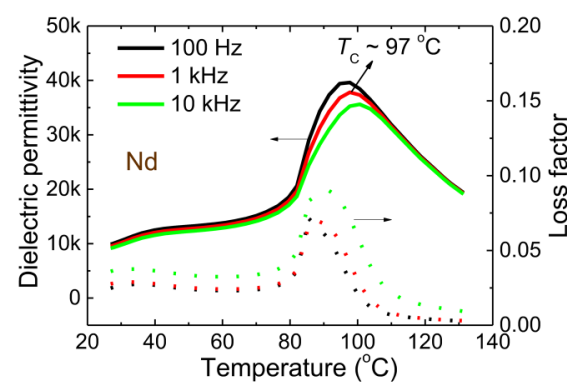

(e)

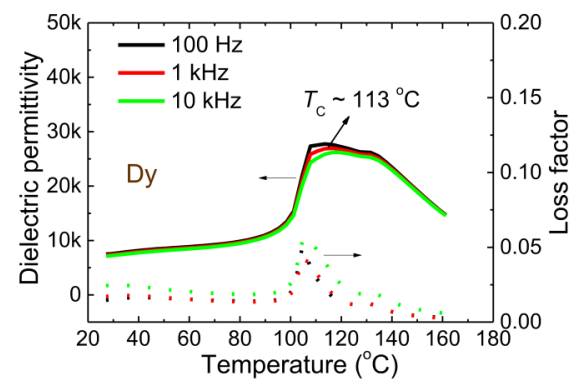

(c)

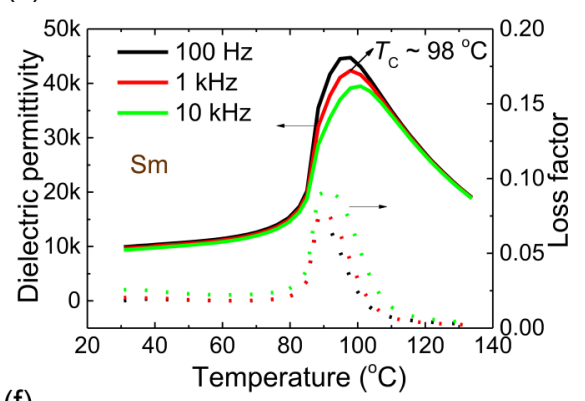

(f)

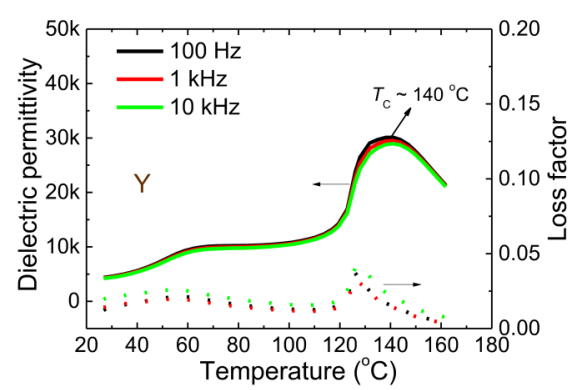

FIG. 3. Temperature dependence of the dielectric constant and loss tangent at three fixed frequencies $(100 \mathrm{~Hz}, 1 \mathrm{kHz}$, and $10 \mathrm{kHz}) \mathrm{for}$ PMN-31PT with 2.5 mol \% dopants: (a) La, (b) Nd, (c) Sm, (d) Gd, (e) Dy, and (f) Y. $T_{C}$ values shown in the inset were recorded at $1 \mathrm{kHz}$.

is directly proportional to the spontaneous polarization $\left(P_{S}\right)$ expressed as $T_{C}=\alpha P_{S}$ ( $\alpha$ is a constant), thus it is reasonable that $A$-site doping lowers the lead concentration and weakens the $\mathrm{Pb}-\mathrm{O}$ hybridization, leading to the reduced polarization and $T_{C}$. There has been an empirical equation showing that $T_{C}$ decreases about $25^{\circ} \mathrm{C}$ per mol \% if rare-earth cation $R^{3+}$ goes to the $A$ site of $\mathrm{Pb}$-based perovskites [22,32]. As shown in Figs. 3(a)-3(c), for $2.5 \mathrm{~mol} \% \mathrm{La}^{3+}, \mathrm{Nd}^{3+}$, and $\mathrm{Sm}^{3+}$ dopants, the value of $T_{C}$ decreases significantly to $98^{\circ} \mathrm{C}, 97^{\circ} \mathrm{C}$, and $98^{\circ} \mathrm{C}$, respectively. Compared with PMN-31PT $\left(T_{C} \sim\right.$ $160^{\circ} \mathrm{C}$ ), the $T_{C}$ of $2.5 \mathrm{~mol} \% \mathrm{La} / \mathrm{Nd} / \mathrm{Sm}$-doped PMN-31PT is decreased by $62-63^{\circ} \mathrm{C}$, following the empirical prediction of $2.5 \times 25^{\circ} \mathrm{C}=62.5^{\circ} \mathrm{C}$. On the contrary, the values of $T_{C}$ for $2.5 \mathrm{~mol} \% \mathrm{Gd}^{3+}$-, $\mathrm{Dy}^{3+}$-, and $\mathrm{Y}^{3+}$-doped PMN-31PT are $107^{\circ} \mathrm{C}, 113^{\circ} \mathrm{C}$, and $140^{\circ} \mathrm{C}$, respectively, being much larger than the predicted $97.5^{\circ} \mathrm{C}$. This indicates that the dopants smaller than $\mathrm{Sm}^{3+}$ cannot entirely occupy the $A$ site of PMN-PT.

To further certify the occupational preference of $R^{3+}$ dopants, the volatilization characteristic of $\mathrm{Pb}^{2+}$ during sintering also reflects the occupational preference of $R^{3+}$ dopants. As shown in Fig. S2 in the SM [26], the gravimetric losses of $\mathrm{La}^{3+}-, \mathrm{Nd}^{3+}$, and $\mathrm{Sm}^{3+}$-doped PMN-31PT ceramics are around $1.9 \%$, which is much larger than that of $\mathrm{Gd}^{3+}-, \mathrm{Dy}^{3+}-$ and $\mathrm{Y}^{3+}$-doped counterparts $(\sim 1.1 \%)$. It is well accepted that in lead-based perovskites, lead is easily volatilized during a high-temperature sintering process because of the low melting point of lead oxide $\left(888^{\circ} \mathrm{C}\right)$. Generally, if the substituent enters the $A$ sublattice, it would replace $\mathrm{Pb}^{2+}$ and cause weight loss [33], which accounts for the more pronounced loss of lead in $\mathrm{La}^{3+}-, \mathrm{Nd}^{3+}$, and $\mathrm{Sm}^{3+}$-doped PMN-PT. Thus, the lower gravimetric losses observed in $\mathrm{Dy}^{3+}$ - and $\mathrm{Y}^{3+}$-doped $\mathrm{PMN}-31 \mathrm{PT}$ again indicates that $\mathrm{Dy}^{3+}$ and $\mathrm{Y}^{3+}$ do not entirely occupy the $A$ site.
Based on the above three experiments, it is safe to conclude that $R^{3+}$ dopants that are larger than $\mathrm{Sm}^{3+}$ prefer occupying the $A$ site in PMN-PT while those smaller ones (e.g., $\mathrm{Gd}^{3+}$, $\mathrm{Dy}^{3+}$, and $\mathrm{Y}^{3+}$ ) prefer occupying $B$-site cations.

After confirming the occupation of the dopants, we carried out first-principles calculations for 0.75PMN-0.25PT (hereafter abbreviated as PMN-25PT) with different $A$-site or $B$-site dopants. The computational details are presented in the SM [26]. Note that PMN-25PT is chosen as it allows a feasible size of the supercell for DFT calculations and the composition is only a few percent lower in PT content than that in PMN29PT and PMN-31PT. A $2 \times 2 \times 2$ supercell with 40 atoms was used to capture the local structural characteristics, as shown in Fig. 4(a). In the supercell, one $\mathrm{Pb}$ atom or one $B$-site atom (see SM for details [26]) is replaced by a dopant [see Figs. 4(b) and 4(c)], which means that the concentration of dopant is $12.5 \%$ in the supercell, being much higher than the average concentration in fabricated ceramics (i.e., <3\%). Thus, the calculation gives a picture depicting the property of the regions around the dopants. $\mathrm{Pb}$ vacancies are not considered in calculations, which may be present due to charge compensation and the volatile nature of $\mathrm{Pb}$; however, such effect on the local structure or polarization is likely to be similar for different rare-earth dopants. Moreover, considering the affordable size of the supercell in DFT computations, it is inappropriate to incorporate two trivalent dopants and one $\mathrm{Pb}$ vacancy in such cell, as the defect concentration would be too high. Figures 4(c) and 4(d) show the lattice parameters and polarizations of the supercell for doped PMN-25PT. For undoped PMN-25PT, the calculations give the lattice parameters with $a=8.075 \AA, b=7.926 \AA$, and $c=8.133 \AA$, showing an orthorhombic-like symmetry with the polar vector being $11^{\circ}$ away from the [101] direction. It should be noted here that the averaged structure of PMN-25PT obtained from experiments 
(a)

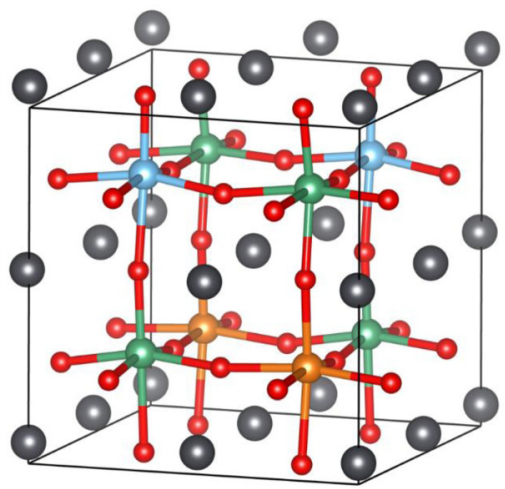

(d)

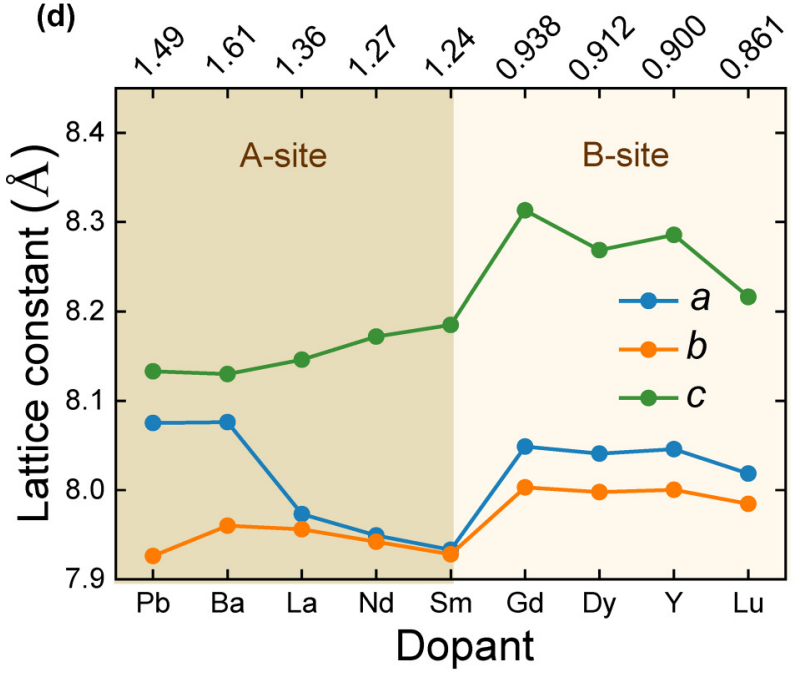

(b)

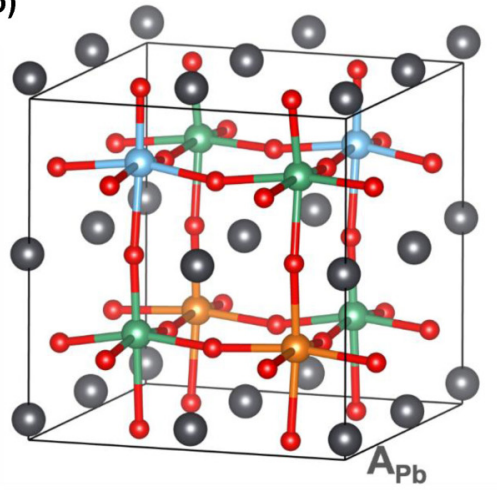

(c)

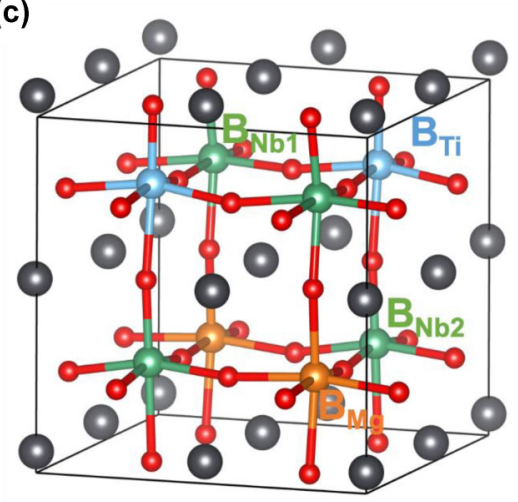

(e)

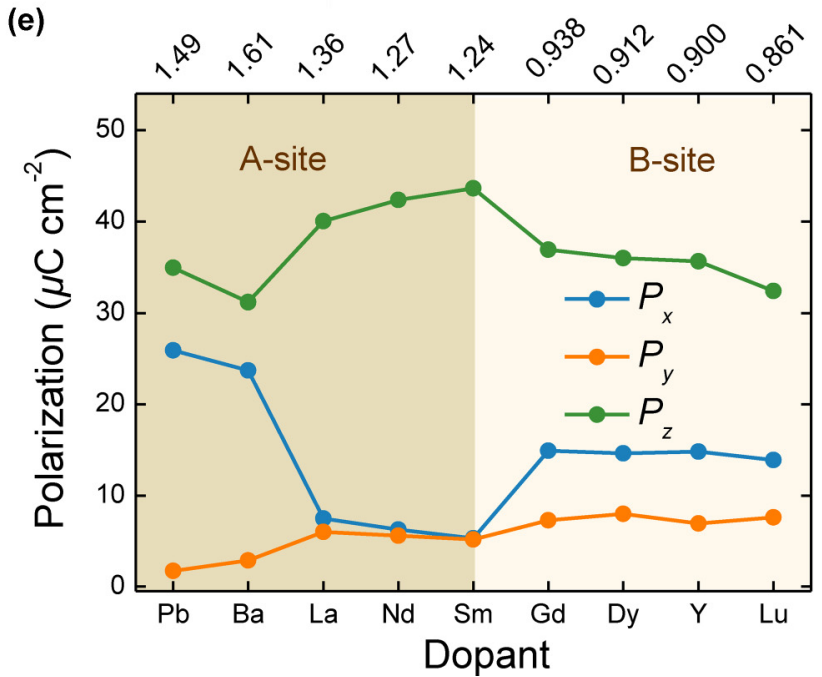

FIG. 4. Structure models, DFT calculated lattice constants, and polarizations for undoped and doped PMN-25PT. (a) A $2 \times 2 \times 2$ supercell with 40 atoms for PMN-25PT; (b) the supercell with $A$-site dopant for one $\mathrm{Pb}$ atom; (c) the supercell with $B$-site dopant for one $\mathrm{Mg}, \mathrm{Nb} 1, \mathrm{Nb} 2$, or $\mathrm{Ti}$ atom. The large gray spheres represent $\mathrm{Pb}$ atoms; the small red spheres denote $\mathrm{O}$ atoms; and the orange, blue, and green spheres are for $\mathrm{Mg}, \mathrm{Nb}$, and Ti atoms, respectively. (c) The lattice parameters $(a, b, c)$, and (d) the polarization components $\left(P_{x}, P_{y}, P_{z}\right)$ of the PMN-25PT and those with dopants. The ionic radius values are from Ref. [34] for $\mathrm{Pb}^{2+}, \mathrm{Ba}^{2+}, \mathrm{La}^{3+}, \mathrm{Nd}^{3+}$, and $\mathrm{Sm}^{3+}$ with coordination number 12 and for $\mathrm{Gd}^{3+}, \mathrm{Dy}^{3+}, \mathrm{Y}^{3+}$, and $\mathrm{Lu}^{3+}$ with coordination number 6 .

is rhombohedral. The difference between the calculated and measured structures is associated with the relatively small size of the $2 \times 2 \times 2$ supercell, which does not include all possible local structures in a practical crystal. In this work, we focus on the relative variation in the local structure with the presence of dopants by first-principles calculations. The calculated volume of PMN-25PT is $520.53 \AA^{3}$ for a $2 \times 2 \times$ 2 supercell, corresponding to $65.07 \AA^{3}$ per unit cell. This value is in good agreement with the XRD results giving $V \sim$ $64.88 \AA^{3}$. By doping rare-earth cations that occupy $A$ sites $\left(\mathrm{La}^{3+}, \mathrm{Nd}^{3+}\right.$, and $\left.\mathrm{Sm}^{3+}\right)$, the lattice parameter $c$ is found to increase while $a$ and $b$ decrease when compared to undoped PMN-PT. Furthermore, the change is more pronounced for the dopants with smaller ionic size. Similar variations are also observed in polarization, i.e., the increase of $P_{z}$ (polarization along the $z$ axis) and the decrease of $P_{x}$ and $P_{y}$ (polarization along the $x$ and $y$ axes, respectively) with decreasing the size of $R^{3+}$ dopants on the $A$ site. These results indicate that the regions on the proximity of dopants are in tetragonal phase for $R^{3+}$-doped PMN-25PT while the regions far away from the dopants are in orthorhombic-like structure. In addition, a smaller size of the doped ions on the $A$ site leads to a larger variation of local structure in PMN-25PT. In contrast, for isovalent $A$-site dopants $\left(\mathrm{Ba}^{2+}\right)$, minimal variation in the lattice parameters is observed as shown in Fig. 4. On the other hand, for the dopants with ionic radii smaller than $\mathrm{Sm}^{3+}$ that substitute $B$-site ions $\left(\mathrm{Gd}^{3+}, \mathrm{Dy}^{3+}\right.$, and $\left.\mathrm{Y}^{3+}\right)$, the variation of polarizations was found to decrease when compared to that of $A$-site $R^{3+}$ dopants. The reason for the minimal impact of $B$-site $R^{3+}$ dopants on local structure when compared to the $A$-site $R^{3+}$ dopants is due to the fact that the spontaneous polarization (the order parameter for ferroelectrics) of PMN$\mathrm{PT}$ is mainly associated with the $\mathrm{Pb}-\mathrm{O}$ hybridization as a result of the lone pair electrons of $\mathrm{Pb}^{2+}$ ions [35].

These first-principles data demonstrate that a smaller ionic size of $A$-site $R^{3+}$ dopants leads to a larger local structural variation in PMN-PT, while the impact of $B$-site $R^{3+}$ dopants on local structure is not as significant as that of $A$-site $R^{3+}$ dopants. Hence, based on both theoretical and experimental results, the reason why Sm-doped PMN-PTs exhibit the highest piezoelectricity when compared to other $R^{3+}$-doped PMNPTs can be explained as below. For PMN-PT, the dopant $\mathrm{Sm}^{3+}$ is the smallest ion that could enter the $A$ site, thus it is the most effective $R^{3+}$ in PMN-PT to induce heterogeneous interfaces 
where polarization and strain are discontinuous. As analyzed by phase-field simulations [22], the competition between the bulk and interfacial energies may lead to a further flattened free-energy landscape and thus an enhanced piezoelectric coefficient $d_{33}$ in a specific temperature range.

In summary, impacts of the valence state and ionic size of the dopants on the local structural heterogeneity and macroscopically piezoelectric properties of PMN-PT have been studied based on first-principles calculations and experimental characterizations. The atomic-scale origin of the ultrahigh piezoelectricity in samarium-doped PMN-PT ceramics was attributed to the fact that $\mathrm{Sm}^{3+}$ is the smallest ion that can totally occupy the $A$ site in PMN-PT. This work is expected to build a bridge between the first-principles calculations and design of high-performance piezoelectric materials.

\section{METHOD}

For DFT calculations, a $2 \times 2 \times 2$ supercell with 40 atoms is used in the calculations to study the local structures of PMN-25PT, which has been successfully employed in previous studies [36-38]. DFT calculations are carried out using the Vienna ab initio simulation package (VASP) [39]. Notably, the $4 f$ electrons of rare earth are not treated as valence electrons, since we are not dealing with magnetism here and the structural properties are not sensitive to $4 f$ electrons [40,41]. Details about the experiments and calculations can be found in the Supplemental Material [26].

\section{ACKNOWLEDGMENTS}

F.L., C.L., and D.L. acknowledge financial support from Natural Science Foundation of China under Grants No. 51922083, No. 51502047, and No. 51502232. B.X. and L.B. acknowledge the ONR for support under Grant No. N0001417-1-2818 and DARPA Grant No. HR0011-15-2-0038 (MATRIX program). S.Z. acknowledges the ONRG for support under Grant No. N62909-18-12168. B.X. also acknowledges support from the startup fund from Soochow University, and support from Priority Academic Program Development (PAPD) of Jiangsu Higher Education Institutions. T.R.S. acknowledges support from ONR.
[1] B. Jaffe, W. R. Cook, and H. Jaffe, Piezoelectric Ceramics (Academic, New York, 1971).

[2] K. Uchino, Piezoelectric Actuators and Ultrasonic Motors (Kluwer Academic, Boston, 1996).

[3] F. Li, S. J. Zhang, Z. Xu, X. Y. Wei, and T. R. Shrout, Adv. Funct. Mater. 21, 2118 (2011).

[4] L. E. Cross, Ferroelectrics 76, 241 (1987).

[5] Y. Saito, H. Takao, T. Tani, T Nonoyama, K. Takatori, T. Homma, T. Nagaya, and M. Nakamura, Nature (London) 432, 84 (2004).

[6] N. Huang, Z. Liu, Z. Wu, J. Wu, W. Duan, B. L. Gu, and X. W. Zhang, Phys. Rev. Lett. 91, 067602 (2003).

[7] H. Liu, J. Chen, L. L. Fan, Y. Ren, Z. Pan, K. V. Lalitha, J. Rödel, and X. R. Xing, Phys. Rev. Lett. 119, 017601 (2017).

[8] S. J. Zhang, F. Li, X. N. Jiang, J. W. Kim, J. Luo, and X. C. Geng, Prog. Mater. Sci. 68, 1 (2015).

[9] S. E. Park and T. R. Shrout, J. Appl. Phys. 82, 1804 (1997).

[10] Z. Wu and R. E. Cohen, Phys. Rev. Lett. 95, 037601 (2005).

[11] X. P. Wang, J. G. Wu, D. Q. Xiao, J. G. Zhu, X. J. Cheng, T. Zheng, B.Y. Zhang, X. J. Lou, and X. J. Wang, J. Am. Chem. Soc. 136, 2905 (2014).

[12] H. Liu, J. Chen, H.B. Huang, L. L. Fan, Y. Ren, Z. Pan, J. X. Deng, L. Q. Chen, and X. R. Xing, Phys. Rev. Lett. 120, 055501 (2018).

[13] M. Ahart, M. Somayazulu, R. E. Cohen, P. Ganesh, P. Dera, H. Mao, R. J. Hemley, Y. Ren, P. Liermann, and Z. G. Wu, Nature (London) 451, 545 (2008).

[14] J. G. Wu, D. Q. Xiao, and J. G. Zhu, Chem. Rev. 115, 2559 (2015).

[15] I. Grinberg, V. R. Cooper, and A. M. Rappe, Nature (London) 419, 909 (2002).

[16] M. Hinterstein, J. Rouquette, J. Haines, P. Papet, M. Knapp, J. Glaum, and H. Fuess, Phys. Rev. Lett. 107, 077602 (2011).
[17] M. H. Zhang, K. Wang, Y. J. Du, G. Dai, W. Sun, G. Li, D. Hu, H. C. Thong, C. L. Zhao, X. Q. Xi, Z. X. Yue, and J. F. Li, J. Am. Chem. Soc. 139, 3889 (2017).

[18] F. Li, S. J. Zhang, T. N. Yang, Z. Xu, N. Zhang, G. Liu, J. J. Wang, J. L. Wang, Z. X. Cheng, Z. G. Ye, J. Luo, T. R. Shrout, and L. Q. Chen, Nat. Commun. 7, 13807 (2016).

[19] Z. Kutnjak, J. Petzelt, and R. Blinc, Nature (London) 441, 956 (2006).

[20] M. E. Manley, D. L. Abernathy, R. Sahu, D. E. Parshall, J. W. Lynn, A. D. Christianson, P. J. Stonaha, E. D. Specht, and J. D. Budai, Sci. Adv. 2, e1501814 (2016).

[21] F. Li, S. J. Zhang, D. Damjanovic, L. Q. Chen, and T. R. Shrout, Adv. Funct. Mater. 28, 1801504 (2018).

[22] F. Li, D. B. Lin, Z. B. Chen, Z. X. Cheng, J. L. Wang, C.C. Li, Z. Xu, Q.W. Huang, X. Z. Liao, L. Q. Chen, T. R. Shrout, and S. J. Zhang, Nat. Mater. 17, 349 (2018).

[23] M. J. Krogstad, P. M. Gehring, S. Rosenkranz, R. Osborn, F. Ye, Y. Liu, J. P. C. Ruff, W. Chen, J. M. Wozniak, H. Luo, O. Chmaissem, Z. G. Ye, and D. Phelan, Nat. Mater. 17, 718 (2018).

[24] H. Takenaka, I. Grinberg, and A. M. Rappe, Nat. Mater. 17, 657 (2018).

[25] F. Li, M. J. Cabral, B. Xu, Z. X. Cheng, E. C. Dickey, J. M. LeBeau, J. L. Wang, J. Luo, S. Taylor, W. Hackenberger, L. Bellaiche, Z. Xu, L. Q. Chen, T. R. Shrout, and S. J. Zhang, Science 364, 264 (2019).

[26] See Supplemental Material at http://link.aps.org/supplemental/ 10.1103/PhysRevB.101.140102 which includes Refs. [1-6], for details about sample preparation, structure and electric properties measurements, first-principles calculations, and results about microstructure, grain distribution, polarization and strain behaviors, and Rietveld refinements.

[27] W. W. Cao and C. A. Randall, J. Phys. Chem. Solids 57, 1499 (1996).

[28] F. Li, Z. Xu, and S. J. Zhang, Appl. Phys. Rev. 1, 011103 (2014). 
[29] S. Körbel, P. Marton, and C. Elsässer, Phys. Rev. B 81, 174115 (2010).

[30] S. C. Abrahams, S. K. Kurz, and P. B. Jamieson, Phys. Rev. 172, 551 (1968).

[31] I. Grinberg and A. M. Rappe, Phys. Rev. B 70, 220101 (2004).

[32] N. Kim, W. Huebner, S. J. Jang, and T. R. Shrout, Ferroelectrics 93, 341 (1989).

[33] L. Wu, C. C. Lee, T. S. Wu, and C. C. Wei, Ferroelectrics 41, 157 (1982).

[34] R. D. Shannon, Acta Crystallogr. A32, 751 (1976).

[35] D. I. Bilc and D. J. Singh, Phys. Rev. Lett. 96, 147602 (2006).
[36] H. Takenaka, I. Grinberg, and A. M. Rappe, Phys. Rev. Lett. 110, 147602 (2013).

[37] H. Takenaka, I. Grinberg, S. Liu, and A. M. Rappe, Nature (London) 546, 391 (2017).

[38] H. Tan, H. Takenaka, C. Xu, W. Duan, I. Grinberg, and A. M. Rappe, Phys. Rev. B 97, 174101 (2018).

[39] G. Kresse and D. Joubert, Phys. Rev. B 59, 1758 (1999).

[40] J. P. Perdew, A. Ruzsinszky, G. I. Csonka, O. A. Vydrov, G. E. Scuseria, L. A. Constantin, X. Zhou, and K. Burke, Phys. Rev. Lett. 100, 136406 (2008).

[41] R. Nakamoto, B. Xu, C. Xu, H. Xu, and L. Bellaiche, Phys. Rev. B 95, 024434 (2017). 